\title{
Past, present and future modally introduced
}

\section{Tomasz Placek ${ }^{1}$ (1)}

Received: 3 January 2019 / Accepted: 22 June 2019 / Published online: 1 July 2019 (c) The Author(s) 2019

\begin{abstract}
We investigate the concepts of past, present, and future that build upon a modal distinction between a settled past and an open future. The concepts are defined in terms of a pre-causal ordering that is determined by the qualitative differences between alternative possible histories. We look what an event's past, present, and future look like in the so-called Minkowskian Branching Structures, one in which histories are isomorphic to Minkowski space-time.
\end{abstract}

Keywords Past $\cdot$ Present $\cdot$ Future $\cdot$ Modality $\cdot$ Theory of relativity

\section{Introduction: a conflict about tenses}

There is an acute conflict between our intuitive notions of past, present, and future, and what current physics has to say about time. ${ }^{1}$ To list the main features of the former (aka manifest time), (i) it assumes a mind-independent tripartite division of the world into past, present, and future. (ii) These three regions are supposed to continuously change as future events turn into present events, and then-into past events. (iii) There is a further difference with respect to openness vs. settledness: the future is seen as open, in contrast to the past that is viewed as settled, or closed. (Whether the present is settled is a subject of a little controversy, with the majority view opting for its being settled, like the past). ${ }^{2}$ Concerning the present, the manifest view of time suggests that (iv) it is global (so any object existing before a given present and living sufficiently long hits upon it), (v) it cannot be repeated, (vi) it is achronal, and (vii) no two presents

\footnotetext{
1 This work is a distant cousin to my paper "Locus for "now" (2011). For their prompts to revise or precisify my earlier position, I am grateful to the audiences of the Bonn Workshop on Time, of the Conference of the Minkowski Institute in Albena, and of the reading group in Philosophy Department of the UC San Diego. I have also benefited considerably from the discussions with Joanna Luc, Jacek Wawer and Leszek Wroński as well as from insightful comments of two anonymous reviewers for this journal. The support of the National Science Centre research Grant Opus 2016/23/B/HS1/00464 is gratefully acknowledged.

2 For a recent characterization of manifest time, see Callender (2017, Ch. 1).
}

Tomasz Placek

tomasz.placek@uj.edu.pl

1 Philosophy Department, Jagielonian University, Grodzka 52, 31-044 Kraków, Poland 
overlap. Although the terms in which we stated the manifest view are somewhat vague, they are precise enough to contradict the positions informed by the current physics of space and time.

Starting with the papers of Rietdijk (1966) and Putnam (1967), there have been arguments showing that relativistic space-times are inimical to the manifest view. ${ }^{3}$ The ensuing debate, which involved Malament (1977), Dieks (1988), Stein (1991), van Benthem (1991), Rakić (1997) and others, led to the following result concerning special relativity $(\mathrm{SR})$ :

$\mathbf{R}$ The following set of premises is logically inconsistent:

(1) The relations used to define co-presence and co-presence itself are invariant with respect to automorphisms of Minkowski space-time,

(2) Co-presence is a transitive relation on Minkowski space-time,

(3) Of two co-present events, one cannot lie within a forward/backward light cone of the other, and

(4) Co-presence is neither the identity nor the universal relation on Minkowski space-time.

To emphasize, $\mathbf{R}$ is an iron-clad result of logic; thus any attempt (including this one) to secure a non-trivial notion of present, that is, the acceptance of (4), entails a violation of at least one of (1)-(3).

Given the above outlined conflict between the manifest image of time and the position informed by current physics, where does the present project lie? Following an intuition associating the future with contingency, we will construct a spatiotemporally extended and frame-independent notion of the present, and, consequently, similar notions of the past and future. That is, out of the seven claims associated with the manifest image, our priority is to save (i). To explain our use of " present", we take this notion to be relative to events: to refer to the present, we fix our attention on a particular event (typically, a salient event like an utterance) and ask what the present of this event is. Our aim is to indicate a part of our world which is a locus for the present (past, future) of an arbitrary event. In other words, we will define the set of events co-present with a given one, as well as the set of future (past) events for an event in question. In doing so, we leave open the matter of the explication of the continuous flow of events, since it clearly lies beyond the scope of the project at hand.

The approach is intended to be conciliatory: on the one hand, we invite the reader to modify her notion of the past, present, and future; taking stock of our construction in Sect. 7 furnishes a list of the modifications required. On the other, we believe that neither special relativity nor general relativity are our ultimate truths, so perhaps one day there will be a theory of space-time amenable to an aspect underlying the future as here constructed, i.e., local indeterminism. ${ }^{4}$

\footnotetext{
3 For a present assessment of Putnam's argument, cf. Dorato (2008).

4 The construction developed here is in some aspects similar to that of Müller's (2006) and to a model I gave at Logica 2002 (unpublished). Some ideas presented here are the fruit of discussions I had with T. Müller in the last decade. I am grateful to him for sharing his insights with me. It also seems to me that Fred Muller once held similar views on loci for the past, present, and future, but I could not find a relevant publication.
} 
The paper is organized as follows. In Sect. 2 we belabor the intuition linking future with contingency. As a further analysis of this intuition requires a framework for discussing modalities and tenses, in Sects. 3 and 4 we sketch the best available framework of this sort, Belnap's (1992) Branching Space-Times (BST) and Minkowskian Branching Structures (MBS's). Section 5 then presents some facts about an event's past, present, and future in MBS's. In Sect. 6 we exhibit the present, past, and future of events in some selected MBS's. Some conclusions are furnished in Sect. 7.

\section{A future-openness link}

\subsection{Some historical remarks}

There is a long tradition that associates the future with open possibilities, the past with settled facts, and the present with a region of passage from possibility to settledness. The view arguably had a proponent in Aristotle (Cael I.12). In more recent times, the idea has been defended by Whitrow (1961, pp. 295-296):

Strict causality would mean that the consequences pre-exist in the premises. But, if the future history of the universe pre-exists logically in the present, why it is not already in the present? If, for the strict determinist, the future is merely "the hidden present", whence comes the illusion of temporal succession? The fact of transition and 'becoming' compels us to recognize the existence of an element of indeterminism and irreducible contingency in the universe. The future is hidden from us - not in the present, but in the future. Time is the mediator between the possible and the actual.

Similar elaborations on this view can be found in Eddington (1949) and (1953). More recently, this position underlies Ellis's models (2006, pp. 1812-1813) of an evolving block universe:

Things could have been different, but second by second, one specific evolutionary history out of all the possibilities is chosen, takes place, and gets cast in stone.

It is not our aim to offer an exegesis of the above pronouncements but it is nevertheless clear that the above quotes link tenses to modalities by these two theses:

1. The Future is genuinely open in the sense that there are several possible ways that the world might develop;

2. As time passes, while some possibilities are getting actualized, the others turn into mere might-have-beens.

In a nutshell, this view links the future to modally understood indeterminism, where this qualification means that alternative evolutions of an indeterministic system are not reducible to modally flat notions, like models of a theory, or Humean laws of nature [for more on the distinction modal vs. non-modal notions of indeterminism, see Placek and Belnap (2012)]. 
The doctrine that the objectivity of the distinction between the past, the present, and the future requires modal indeterminism has been vigorously opposed. ${ }^{5}$ But no friends or foes of the doctrine have belabored the underlying association between future and contingency to a point of stating it with a rigor that would make the association amenable to formal treatment and then-to rigorous criticism. It is precisely this first task to which we now turn.

\subsection{Belaboring the future-openness link}

There are two intuitions that seem relevant to constructing the past, present, and future from modal notions: the settledness of the past intuition, and the openness of the future intuition. To clarify them, we turn our attention to how we speak about future events, and consider what explanations of futurity in modal terms are acceptable. So we will investigate schematic explanations of the form "event $f$ belongs to the future of event $e$ because ...". Once we find an acceptable explanation of this kind, we will turn it into the truth-conditions for " $f$ belongs to the future of $e$ ", and research the consequences of these truth-conditions in later sections. We will focus on concrete token-level events, like the Summer Solstice in 2019, which do not and (arguably) cannot repeat in a given course of events. Token-level events can have a modal aspect as well, witnesses by phrases like "That event could have occurred earlier (in a a slightly different location/in a slightly different way) than it actually occurred". David Lewis (1986, 195nn) called such events "non-fragile", while Belnap (2005) called them "disjunctive". A distinctive feature of a non-fragile/disjunctive event is that although it cannot be repeated in any given history (so it is a token-level event), it might have different pasts, so it might belong to more than one history. In everyday modal talk we arguably refer to non-fragile/disjunctive events. ${ }^{6}$

To investigate our intuitions, consider two concrete events that appear to be good candidates for one being in the future of the other: the Summer Solstice in $2019(s)$ and a rainy sunrise on Nov 20, 2018 in Del Mar $(r)$. The first intuition sees the past as settled. That is, although before that particular sunrise in Del Mar things could go differently (it might be rainy, and it might be sunny, and it might be foggy, etc.), from the perspective of a future event, like $s$, it is settled (fixed, inevitable) that there was this rainy sunrise. The settledness of the past intuition suggests the following schematic explanation SP:

SP Event $s$ belongs to the future of event $r$ because at event $s$ it is settled that $r$ has happened.

The openness of the future intuition is more elusive; we begin tentatively with this proposal:

A $s$ belongs to the future of $r$ because it is not settled at $r$ that $s$ will occur.

\footnotetext{
5 See e.g., Gale's (1963) attempt to rebut Whitrow's and Eddington's arguments.

6 To give a real-life example, in wrestling circles it was hotly debated in Winter 2019 whether Becky Lynch's entrance could have been improved. No-one argued that Lynch's entrance was what it was, so it could not have been improved. An argument of this kind would suggest that Lynch's entrance is understood as a fragile event.
} 
Our schematic explanation A seems wrong, however. Perhaps this is overly optimistic, but I am inclined to think that no matter how the world evolves from its conditions in Nov 2018, there is the 2019 solstice in each of its possible evolutions. Answer A sound bad because in this case either the explanans is false, or $s$ does not lie in the future of $r$. Let us therefore try another one:

B $s$ belongs to the future of $r$ because a way $s$ will occur is not settled at $r$.

Although answer $\mathbf{B}$ does not look immediately incorrect if applied to Summer Solstice 2019 , it is still counter-intuitive. To see this, think of your grandfather's Swiss watch (mechanical, almost perfect, always wound); suppose it sits in an isolating contraption, and ask yourselves if it is already settled how it will signal tomorrow's noon. My intuition would be that this is already settled, no matter what its surroundings are. Like with answer $\mathbf{A}$, in this case either the explanans is false, or $s$ does not lie in the future of $r$.

The moral is that we need to accommodate the surroundings of $s$, which is what proposal $\mathbf{C}$ does. It commends itself as being sufficiently weak, while still linking future to contingency.

C $s$ belongs to the future of $r$ because before $s$ there is an event and some aspect of it that is not settled at $r$.

In other words, for $s$ to belong to the future of $r$, one need some, however small, contingency, like Heads or Tails, to occur before $s$ (but not necessarily after $r$ ). On this proposal, a small but properly located contingency makes a tomorrow's event involving your grandfather's watch belong to the future of your reading these words, no matter how well is the watch isolated. Somewhat jocularly, this answer seems to make you and me the creators of the future: if in fear that a tomorrow's process is not in our future, flip a coin, do a Stern-Gerlach experiment, or the like. The joke, of course, is that if the results of a coin toss or a Stern-Gerlach experiment as well as our "decision" to perform them are predetermined, it is not in our power to create the future. But if there is no such pre-determination, no action on our side is needed to create the future, as it is already there.

Since the schematic explanations $\mathbf{C}$ and $\mathbf{S P}$ of futurity seem acceptable, at least as far as modal explanations go, we thus turn them into truth-conditions for the sentence " $f$ is in the future of $e$ ":

$\mathbf{S} f$ is in the future of $e$ iff it is settled at $f$ that $e$ has happened, and there is some event $e^{\prime}$ weakly before $f$ and a subject matter $A$ such that at $e$ it is contingent that $A$ obtains at the location of $e^{\prime}$.

Here "weakly before" is understood as "before or identical to". But "before" in our use calls for further attention, as it relates particular events that are not necessarily from the actual history. In contrast to Obama's presidency occurring before Trump's (in our history), we will say that a particular coin toss occurs before $(\leqslant)$ each of its alternative possible outcomes: the toss is before tails up and before heads up. We will read $e \leqslant e^{\prime}$ in English as " $e$ can really happen after $e$ ". The ordering is thus $B$-theoretic, but it has a modal aspect. Note that incomparability by $\leqslant$ of two events can indicate two things: the events belong to one history but none can really happen after the other (because 
they are space-like related), or there is no possible history to which they belong. In the context of Special Relativity, the ordering is to extend the Minkowski ordering $\leqslant_{M}$ of $\mathfrak{R}^{4}$, defined in Eq. 1, to modal contexts.

Our plan is to make $\mathbf{S}$ precise and then define the future of $e$, for any given event $e$. By grasping a concept of the future, a characterization of the past and the present follows naturally. We will define events in the past of event $e$ as those events from which perspective $e$ is in the future. Having had the notions of "events in the past of $e$ " and "events in the future of $e$ ", we will declare that event $e^{\prime}$ is in the present of $e$ iff $e$ and $e^{\prime}$ share a possible history and $e^{\prime}$ is neither in the past nor in the future of $e$. Note that on this construal, an event's past, present, and future are sets of events, that is, they are event-like concepts. They are not location-like concepts; consequently, we will not discuss in this framework what the present of a given spatiotemporal location is.

To reflect on what resources are needed to analyze $\mathbf{S}$, apart from particular events, $e, e^{\prime}$, and $f$ related by the pre-causal relation $\leqslant, \mathbf{S}$ invokes spatio-temporal locations of events, a subject matter (which will be naturally rendered as a formula of a specified language), modal notions of contingency and settledness; further $\mathbf{S}$ relativizes correctness of a sentence to spatio-temporal events. Thus, to analyze $\mathbf{S}$ we need a semantics for a language containing tense operators ("it will be the case that", "it was the case that"), modal operators ("it is still possible that", "it is already settled that"), and location operators ("at location $(x, t)$ it is so that"); furthermore, the evaluation points in this semantics should include spatio-temporal events. In other words, we need a rigorous indexical framework representing space-time and local modalities. Moreover, to obtain traction with the space-times of physics, some structures definable in that framework (histories) should be isomorphic to Minkowski space-time, or space-times of General Relativity.

The news is that there is on a market a rigorous framework precisely of this kind: branching space-times (BST) of Belnap (1992); as it is axiomatic, it gives us an extra control over our constructions. ${ }^{7}$ The rest of this paper is thus somewhat automatic. We will translate schema $\mathbf{S}$ into a more regimented language amenable to the BST analysis. We then look into BST models to learn what object it is, the totality of events lying in the future of a specified event. We will focus on relevant models of BST, Minkowskian Branching Structures (MBS's) in which possible histories are isomorphic to Minkowski space-time. There we could discern particular regions for present, past, and future, so we can relate these findings to the mentioned debate about tenses and Special Relativity.

\section{Models of BST}

The basic insight of branching theorists, one which we owe to Arthur Prior, is that sentences are evaluated as true or false in event-history pairs. This can be seen as adding more structure to Tarski's notion of a sentence being true or false in a model.

\footnotetext{
7 BST is a development of an earlier theory of branching time (BT) of Prior (1967). BT theory in turn was suggested in S. Kripke's letter to Prior (dated September 3, 1958, unpublished); later it was worked out in Thomason (1970).
} 
In a branching-style framework, a sentence is true in a model at an event-history pair $e / h$-this notation is to indicate that $e \in h$. In this spirit, we have the following:

$$
e / h_{1} \models \text { Will: } H \text { but } e / h_{2} \not \neq W i l l: H \text {, }
$$

where a sentence $H=$ "this flipped coin is landing heads up", $e$ is the event of tossing this particular coin, and $h_{1}, h_{2}$ are histories, each containing the toss, with heads landing up in $h_{1}$ and tails landing up in $h_{2}{ }^{8}$

A structure of BST, $\langle W, \leqslant\rangle$, is a non-empty partially ordered set of possible point events ordered by a pre-causal relation, subject to some postulates. (For the postulates, see "Appendix"). Histories in $\langle W, \leqslant\rangle$ are identified with particular (upward directed) subsets of $W$, in accord with what might be called "the later witness criterion". Namely, if two events can occur before a third event, then all the three are co-possible, i.e., they share a possible history. A BST structure $\langle W, \leqslant\rangle$, with an interpretation $\mathcal{I}$ added, is a semantic model $\langle\langle W, \leqslant\rangle, \mathcal{I}\rangle$ for a propositional language with tense operators and modal operators. Interpretation $\mathcal{I}$ is a function $\mathcal{I}:$ Atoms $\mapsto \mathcal{P}(W)$, where Atoms is the set of atomic formulas. ${ }^{9}$

It is understood that atomic formulas of this language have the form: "Here-andnow there is property $X$ ". Turning to truth-conditions that recursively define truth at a point of evaluation, we list a few examples. [(To avoid lengthy notation, we abbreviate the point of evaluation $\langle\langle\langle W, \leqslant\rangle, \mathcal{I}\rangle, e / h\rangle$ to $e / h$. For more information on BST semantical models, cf. Belnap (2007)].

$e / h \models A$ iff $e \in \mathcal{I}(A)$ for $A$ an atomic formula;

$e / h \models \neg \varphi$ iff it is not the case that $e / h \models \varphi$;

$e / h \models$ Will: $\varphi$ iff there is $e^{\prime}>e$ s.t. $e^{\prime} / h \models \varphi$;

$e / h \models$ Was: $\varphi$ iff there is $e^{\prime}<e$ s.t. $e^{\prime} / h \models \varphi$;

$e / h \models$ Poss : $\varphi$ iff there is history $h^{\prime}$ s.t. $e \in h^{\prime}$ and $e / h^{\prime} \models \varphi$;

$e / h \models S e t t: \varphi$ iff for every history $h^{\prime}$, if $e \in h^{\prime}$ then $e / h^{\prime} \models \varphi$.

Note that in the last two clauses, since we quantify over histories on their right-hand sides, the reference to history on the left-hand sides is redundant. We will thus write $e \models$ Poss : $A$ and $e \models S e t t: A$ instead of $e / h \models P o s s: A$ and $e / h \models S e t t: A$, resp.

Some (but not all) BST structures admit a further feature: space-time locations (stlocations for short). A set Loc of st-locations for BST model $\langle W, \leqslant\rangle$ is a partition of $W$ that is conservative with respect to ordering $\leqslant-$ cf. Müller (2005). St-location is a relativistic counterpart of our everyday thinking of what would happen at the time and in the spatial location of a given event, if things went differently at some junction in the past. Note that we have thus arrived at the distinction between event (i.e., an element of $W$ ) and st-location of an event (an element of a particular partition Loc of $W)$. To denote the st-location of event $e$, we will write $\operatorname{loc}(e)$.

In what follows, we need to consider sentences of the form "At st-location $x$ it is $\varphi$ ", like "The value of electromagnetic field at $x$ is such-and-such." The truth conditions

\footnotetext{
8 Note another feature: the language is indexical and tensed.

9 A more general option is to take an interpretation to assign sets of pairs $\langle e, h\rangle$ (where $e \in h$ ) to atomic formulas, but we use here a simpler option. For a discussion of this matter, see Belnap and Müller (2014).
} 
for such sentences can only be formulated with respect to a BST model with set Loc of st-locations: $:^{10}$

$$
e / h \models A t_{x}: \varphi \text { iff } \exists e^{\prime}: e^{\prime} \in h \cap x \wedge e^{\prime} / h \models \varphi \text {, where } x \in L o c \text {. }
$$

To comment on the evaluation in BST, sentences are always evaluated in pairs $e / h$. If the sentence's main operator is a tense operator, we keep $h$ fixed, and search for $e^{\prime}$ on $h$ s.t. at $e^{\prime} / h$ the sub-formula is true. If the main operator is a modal operator, we keep $e$ fixed and quantify over histories passing through $e$. And, if the main operator is $A t_{x}$, we keep $h$ fixed and locate a unique element $e^{\prime}$ of the intersection $x \cap h$; we evaluate then the sub-formula at $e^{\prime} / h$. (By the construction of Loc, any $x \in L o c$ and any history $h$ intersect at a single event).

We are now able to formulate schema $\mathbf{S}$ within a language amenable to BST analysis:

Definition 1 (future) An event $f$ belongs to the future of event $e, f \in \operatorname{Future}(e)$, iff there is event $e^{\prime}$ and an atomic formula $A$ such that

1. $e^{\prime} \leqslant f$ and

2. $e \models$ Poss : At $t_{\text {loc }\left(e^{\prime}\right)}: A$ and

3. $e \models$ Poss : At $t_{\text {loc }\left(e^{\prime}\right)}: \neg A$, and

4. for every history $h$, if $f \in h$, then $e \in h$.

To explain, the first clause requires that a witness $e^{\prime}$ for $f$ belonging to the future of $e$ occur weakly before $f$. The meaning of clauses (2) and (3) is that from the perspective of $e$, it is contingent whether $A$ is true at location of the witness $e^{\prime}$. The last clause encapsulates SP. Note that we restrict our attention to atomic formulas: other formulas might have traces of futurity/pastness or modalities which jeopardize this definition.

We end this section with the definition of the past of $e$ and the present of $e$, both phrased in terms of Future $\left(e^{\prime}\right)$, for some $e^{\prime} \in W$ :

Definition 2 (past and present) Event $e^{\prime}$ belongs to the past of event $e, e^{\prime} \in \operatorname{Past}(e)$, iff $e \in$ Future $\left(e^{\prime}\right)$.

Event $e^{\prime}$ belongs to the present of event $e, e^{\prime} \in \operatorname{Present}(e)$, iff $e^{\prime} \notin \operatorname{Past}(e), e^{\prime} \notin$ Future (e), and there is a history $h$ such that $e, e^{\prime} \in h$.

Note that clause (4) of Definition 1 implies that if $e \in$ Future $\left(e^{\prime}\right)$, then $e, e^{\prime}$ share some history. Similarly, if $e \in \operatorname{Past}\left(e^{\prime}\right)$, then $e, e^{\prime}$ share some history. For the definition of $\operatorname{Present}\left(e^{\prime}\right)$, the requirement of sharing a history has to be added separately. Apart from this addition, the definition characterizes the Present purely negatively, as neither Past nor Future. Although (we think) this is a hardly a contestable characterization, one might ask for more. After all, human agents seem to be directly apprehending their nows, so a positive description of distinctive features of the nows should be possible. Yet we are skeptical: it looks as if our direct contact with our presents has made us blind to their descriptive features. So we rest satisfied with the negative definition given above.

\footnotetext{
10 If all histories in a BST model share the same structure of locations (which is the case for MBS's we consider next) there is no obstacle to define tense operators in terms $A t_{x}$ operators and the ordering of locations. We nevertheless follow here the usual exposition of BST because in Conclusions we point to an odd contrast between tense operators and metaphysical tenses investigated in this paper.
} 


\section{Minkowskian branching structures (MBS)}

Although the above definitions adequately (we believe) capture our informal statement of $\mathbf{S}$, they do not permit us to "see" what the future, and hence the past and the present, of an event are. This is a consequence of the generality of BST, which leaves it open what BST histories exactly are, as long as they are maximal upward directed subsets of a base set. Thus, to address the "see" question, we need to make it relative to a specific concept of space-time, and then consider such BST models, in which histories are isomorphic to the space-time in question. We will investigate the problem for Minkowski space-time.

We will now turn to Minkowskian branching structures, i.e., BST structures in which every history is isomorphic to Minkowski space-time. ${ }^{11}$ To begin informally, a possible Minkowskian world can be thought of as Minkowski space-time plus physical content. The content can be represented by the attribution of "point properties" (typically, strengths of physical fields), i.e., a function from $\mathfrak{R}^{4}$ to $\mathcal{P}(P)$, where $P$ is the set of point properties. To arrive at a modal aspect, as exemplified for instance in saying "It is $\varphi$ at $x \in \mathfrak{R}^{4}$, but it could be $\psi$ there", we need a system of such physical contents. A system of this sort is represented by a property attribution $F: \Re^{4} \times \Sigma \rightarrow \mathcal{P}(P)$, where $\Sigma$ is the set of labels for scenarios.

Since we haven't (yet) imposed any restrictions on property attributions, we should expect that they sometimes produce strange patterns of properties. In an attempt to arrive at BST structures, we thus single out the class of "proper" property attributions.

We shall put our requirement informally first: for $F$ to be a proper property attribution, we require that every two scenarios $\sigma, \eta \in \Sigma$ are qualitatively different somewhere and if they are different at some point, there is a special point $c \in \Re^{4}$ below this point ( $c$ is called splitting point for $\sigma$ and $\eta$ ). Its special character consists in that (1) $\sigma$ and $\eta$ agree at and below $c$, and that (2) for a point $x$ above $c$, no matter how close $x$ is to $c$, there is always an even closer point above $c$ at which $\sigma$ and $\eta$ disagree in content. ${ }^{12}$ Note that while postulating a complete qualitative agreement at and below $c$, we do not require a complete disagreement above $c$; we readily permit that over large regions above $c$ the scenarios are qualitatively the same-as long as they are different at locations arbitrarily close to $c$ and above $c$. The locutions "above" and "below" refer here to the so-called Minkowskian ordering $\leqslant_{M}$ of $\mathfrak{R}^{4}$ :

$$
x \leqslant_{M} \quad y \text { iff } \sum_{i=1}^{3}\left(x^{i}-y^{i}\right)^{2} \leq\left(x^{0}-y^{0}\right)^{2} \text { and } x^{0} \leq y^{0},
$$

with a resulting strict ordering $<_{M}$ defined in a usual way (above $\leq$ denotes the standard ordering of reals). The relation of being space-like related (henceforth, SLR) is also typically defined: two points are SLR iff they are incomparable by $\leqslant_{M}$. Putting the above explanation in symbols, we have the following:

\footnotetext{
11 MBS's have been investigated by Müller (2002), Wroński and Placek (2009) and Placek and Belnap (2012).

12 The background of the requirement is the density of a BST ordering, which is a BST postulate.
} 
Definition 3 A property attribution $F: \mathfrak{R}^{4} \times \Sigma \rightarrow \mathcal{P}(P)$ is proper iff for every $\sigma, \eta \in \Sigma(\sigma \neq \eta)$ there is $x \in \Re^{4}$ such that

$$
F(x, \sigma) \neq F(x, \eta) \text {, and }
$$

(for every $\left.x \in \mathfrak{R}^{4}\right)$ if $F(x, \sigma) \neq F(x, \eta)$, then there is $c \in \mathfrak{R}^{4}$ such that $c<_{M} x$ and

$$
\begin{aligned}
& \forall z \in \mathfrak{R}^{4}\left(z \leqslant_{M} c \rightarrow F(z, \sigma)=F(z, \eta)\right) \text { and } \\
& \forall x^{\prime} \in \mathfrak{R}^{4}\left(c<_{M} x^{\prime} \rightarrow \exists y \in \mathfrak{R}^{4}\left(c<_{M} y<_{M} x^{\prime} \wedge F(y, \sigma) \neq F(y, \eta)\right)\right) .
\end{aligned}
$$

All the points of $\Re^{4}$ that satisfy conditions 3-4 constitute what we call the set $S_{\sigma \eta}$ of splitting points for $\sigma$ and $\eta$. The meaning of Eq. 4 is that within any maximal chain in $\Re^{4}$ passing through a splitting point $c$, there is a sequence of points above $c$ and convergent to $c$, every element of which has differing property attributions in $\sigma$ and in $\eta$. From the definition of proper property attribution one can deduce some desired properties of sets of splitting points. ${ }^{13}$

To state them, it is useful to distinguish special subsets of $\Re^{4}$, thought of as regions of no qualitative difference of histories, and defined as ${ }^{14}$

$$
R_{\sigma \eta}:=\left\{x \in \mathfrak{R}^{4} \mid \neg \exists c\left(c<_{M} x \wedge c \in S_{\sigma \eta}\right)\right\} \text { for } \sigma, \eta \in \Sigma .
$$

Fact 4 Assume that $F: \mathfrak{R}^{4} \times \Sigma \rightarrow \mathcal{P}(P)$ is a proper property attribution. Then:

1. $\sigma \neq \eta \rightarrow S_{\sigma \eta} \neq \emptyset$;

2. $S_{\sigma \eta}=S_{\eta \sigma}$;

3. $\forall c, c^{\prime} \in S_{\sigma \eta}\left(c \neq c^{\prime} \rightarrow c S L R c^{\prime}\right)$;

4. $x \in R_{\sigma \eta} \rightarrow F(x, \sigma)=F(x, \eta)$; and

5. $\forall \sigma, \eta, \gamma \in \Sigma R_{\sigma \eta} \cap R_{\eta \gamma} \subseteq R_{\sigma \gamma}$.

Clearly, $\Sigma$ is not the set of BST histories, and $\leqslant_{M}$ is not a BST ordering-they do not satisfy the BST postulates. (But there is a perfect match between the two orderings if an MBS contains one history only-see Eq. 7). To produce a BST structure, we need to construct these latter notions, showing that they satisfy BST postulates. In this task, we follow Müller's (2002) construction, to which the reader should turn to for more information. First, we define relation $\equiv$ on $\Re^{4} \times \Sigma::^{15}$

$$
x \sigma \equiv y \eta \text { iff } x=y \text { and } x \in R_{\sigma \eta} .
$$

Provably $\equiv$ is an equivalence relation on $\mathfrak{R}^{4} \times \Sigma$. Next, we define a BST event as an equivalence class with respect to $\equiv$, that is

$$
\{y \eta \mid y \eta \equiv x \sigma\}:=[x \sigma]
$$

\footnotetext{
13 We follow here the construction of Placek and Belnap (2012), which is more "physical" than the others, since it derives BST structures from property attributions. Apart from the proper property attributions, these authors assume a topological postulate and a condition on chains of splitting points.

14 These are not maximal regions of qualitative similarity, as some regions above a splitting point can be similar as well.

15 To avoid eyestrain, we write $x \sigma$ rather than $\langle x, \sigma\rangle$.
} 
$\sigma$

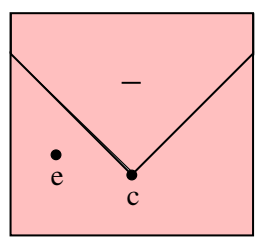

$\sigma$

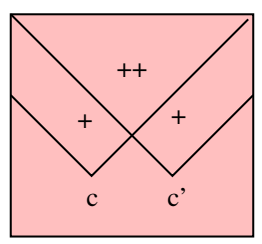

$\eta$

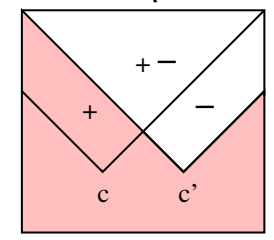

$\eta$

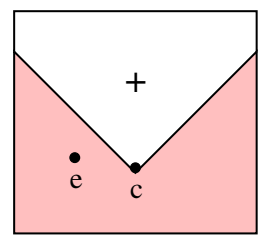

$\tau$

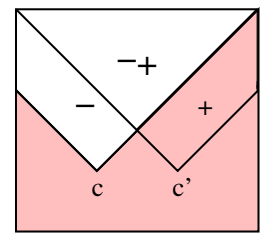

$\gamma$

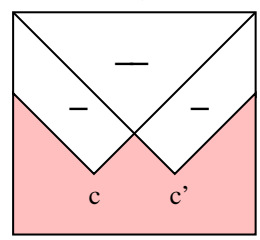

Fig. 1 Top: an MBS with one splitting point and two histories. Bottom: an MBS with two splitting points and four histories. Shadowed regions indicate the intersection of a given history with a reference history $\sigma$. In contrast to this diagram, BST axioms do not dictate that such two splitting points require four histories

A BST ordering is defined as follows:

$$
[x \sigma] \leqslant[y \eta] \text { iff }[x \sigma]=[x \eta] \wedge x \leqslant M \quad y .
$$

Importantly, it turns out that $\Sigma$ is indeed a set of labels for histories, as every BST history is of the form: $h_{\sigma}=\left\{[x \sigma] \mid x \in \mathfrak{R}^{4}\right\}$ for $\sigma \in \Sigma$. It follows that the MBS ordering $\leqslant$, if restricted to a single history, coincides with the Minkowski ordering $\leqslant_{M}$, as we clearly have:

$$
[x \sigma] \leqslant[y \sigma] \text { iff } x \leqslant \leqslant_{M} y .
$$

Moreover, given that a property attribution is proper and an additional postulate is satisfied, ${ }^{16}[x \sigma]$ is a maximal element in the overlap of two histories $h_{\sigma}$ and $h_{\eta}$ iff $x$ is a splitting point for these histories, i.e., $x \in S_{\sigma \eta}$. The construction should finish with proofs that the resulting structure is indeed a BST model. ${ }^{17}$

Having done all this dry and formal work, we will now avail ourselves of a picture as an interlude. Figure 1 illustrates two Minkowskian Branching Structures, the first with two histories and one splitting point, and the second-with four histories and two splitting points. The shaded area indicates where a given history overlaps with the first history.

Formally speaking, an MBS is a triple $\langle\Sigma, P, F\rangle$, where $\Sigma$ is a set of labels for scenarios, $P$ is a set of point properties, and $F$ is a proper property attribution. A merit of this construction is that $\langle\Sigma, P, F\rangle$ provides a natural semantic model for a propositional language with tense operators and modal operators, and whose atomic sentences have the form:

\footnotetext{
16 The postulate is: every convergent sequence in a set $S_{\sigma \eta}$ is convergent to an element of $S_{\sigma \eta}$-cf. Placek and Belnap (2012).

17 For proofs we refer the reader to Placek and Belnap (2012), or Müller (2002) and Wroński and Placek (2009).
} 
It is $\psi$ here-and-now,

where $\psi \in P$. Furthermore, the proper property attribution $F$ determines interpretation function $\mathcal{I}$ in the following manner:

$[x \sigma] \in \mathcal{I}(A)$ iff $\psi \in F(x, \sigma)$, where $A=$ "It is $\psi$ here-and-now".

The BST truth conditions for tense and modal operators can be readily reformulated in the MBS framework. For a point of evaluation we take $\langle\langle\Sigma, P, F\rangle,[x \sigma] / \sigma\rangle$, which we abbreviate as $[x \sigma] / \sigma$. Location $\operatorname{loc}([x \sigma])=x$, of course. As an example, we give below the truth conditions for Poss and $A t_{y}\left(y \in \Re^{4}\right)$ :

$[x \sigma] / \sigma=$ Poss : $B$ iff there is $\eta \in \Sigma$ such that $[x \sigma]=[x \eta]$ and $[x \eta] / \eta \models B$

$[x \sigma] / \sigma \models A t_{y} B$ iff $[y \sigma] / \sigma \models B$, where $y \in \mathfrak{R}^{4}$.

Since in the clause for Poss the reference to label $\sigma$ after the stroke is redundant, we will write $[x \sigma] \models$ Poss : $B$ for $[x \sigma] / \sigma \models$ Poss : $B$.

With this whole machinery in place, we will now translate Definition 1 into the language of MBS's and then prove some properties of the past, present, and future in this framework. Below we write $H_{x \sigma}$ for the set of labels for those histories to which $[x \sigma]$ belongs, i.e., $H_{x \sigma}:=\{\eta \in \Sigma \mid[x \sigma]=[x \eta]\}$.

Definition 5 (future, MBS-style) An event $f=[x \sigma]$ belongs to the future of event $e=[y \eta],[x \sigma] \in$ Future $([y \eta])$, iff there is event $e^{\prime}=\left[z \sigma^{\prime}\right]$ and an atomic formula $A$ such that

1. $\left[z \sigma^{\prime}\right]<[x \sigma]$ and

2. $[y \eta] \models$ Poss : $A t_{z}: A$ and

3. $[y \eta] \models$ Poss : $A t_{z}: \neg A$, and

4. $H_{x \sigma} \subseteq H_{y \eta}$.

The past and the present of a given event are defined analogously as in Definition 2.

\section{Past, present and future: results}

In this section we will prove a few facts about past, present, and future in MBS's and discuss their features.

Modality first! We observe that the past, present, and future of a given event are fully characterized by simple facts about the inclusion of possible histories. This means that the three notions are characterized in modal terms only.

Fact 6 1. $[x \sigma] \in$ Future $([y \eta])$ iff $H_{x \sigma} \subsetneq H_{y \eta}$;

2. $[x \sigma] \in \operatorname{Past}([y \eta])$ iff $H_{y \eta} \subsetneq H_{x \sigma}$;

3. $[x \sigma] \in \operatorname{Present}([y \eta])$ iff there is $\gamma \in \Sigma$ such that $[x \sigma]=[x \gamma]$ and $[y \eta]=[y \gamma]$ and (i) $H_{y \gamma}=H_{x \gamma}$ or (ii) $H_{y \gamma} \backslash H_{x \gamma} \neq \emptyset$ and $H_{x \gamma} \backslash H_{y \gamma} \neq \emptyset$;

4. Future $([y \eta])$ is closed upward, i.e., if $[x \sigma] \in$ Future $([y \eta])$ and $[x \sigma] \leq\left[x^{\prime} \sigma^{\prime}\right]$, then $\left[x^{\prime} \sigma^{\prime}\right] \in$ Future $([y \eta])$; 
5. Past $([y \eta])$ is closed downward, i.e., if $[x \sigma] \in \operatorname{Past}([y \eta])$ and $\left[x^{\prime} \sigma^{\prime}\right] \leq[x \sigma]$, then $\left[x^{\prime} \sigma^{\prime}\right] \in \operatorname{Past}([y \eta])$.

Proof $(1) \Rightarrow .(\star) H_{x \sigma} \subseteq H_{y \eta}$ is just clause (4) of Definition 5. To prove the strict inclu-

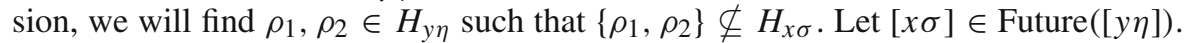
By clause (1) of the same Definition 5, there is a witness $\left[z \sigma^{\prime}\right] \leqslant[x \sigma]$, which implies $z \leqslant_{M} x$ and $\left[z \sigma^{\prime}\right]=[z \sigma]$. Clause (2) and (3) of this Definition then imply, by the truth-conditions (9), that there are $\rho_{1}, \rho_{2} \in \Sigma$ and atomic formula $A$ s.t. ( $\dagger$ ) $\left[z \rho_{1}\right]=A$ and $\left[z \rho_{2}\right]=\neg A$, and $[y \eta]=\left[y \rho_{1}\right]=\left[y \rho_{2}\right]$, so (†) $\rho_{1}, \rho_{2} \in H_{y \eta}$. Observe next that $(\dagger)$ means that $F\left(z \rho_{1}\right) \neq F\left(z \rho_{2}\right)$. Definition 3 then requires the existence of a particularly located splitting point, $c \in S_{\rho_{1} \rho_{2}}$ s.t. $c<_{M} z$, so $c<_{M} x$ (since $[z \sigma] \leqslant[x \sigma]$ ). Hence $x \notin R_{\rho_{1} \rho_{2}}$. Thus, by Eqs. 5 and $6,\left[x \rho_{1}\right] \neq\left[x \rho_{2}\right]$, so it is impossible that both $[x \sigma]=\left[x \rho_{1}\right]$ and $[x \sigma]=\left[x \rho_{2}\right]$. Hence $\left\{\rho_{1}, \rho_{2}\right\} \nsubseteq H_{x \sigma}$. This implies, taken together with ( $\ddagger)$ and $(\star)$, the strict inclusion $H_{x \sigma} \subsetneq H_{y \eta}$.

$(1) \Leftarrow$. RHS implies that there is $\gamma \in \Sigma$ s.t. $[x \sigma] \neq[x \gamma]$ but $[y \eta]=[y \gamma]=[y \sigma]$. By Eqs. 5 and 6, there is $c \in S_{\sigma \gamma}$ s.t. $c<_{M} x$. Thus, by clause (4) of Definition 3, there is $z$, with $(\dagger) c<_{M} z<_{M} x$, such that $F(z \sigma) \neq F(z \gamma)$, hence for some atomic $A,[z \sigma] \models A$ and $[z \gamma] \models \neg A$ (or vice versa). Since $\sigma, \gamma \in H_{y \eta}$, we get $[y \eta] \models$ Poss : $A t_{z}: A$ and $[y \eta] \models$ Poss : At $: \neg A$, so clauses (2) and (3) of Definition 5 are satisfied. Clause (4) follows from RHS, and clause (1) is implied by $(\dagger)$.

(2) Immediate from (1).

(3) From (1) and (2) above, and by the requirement that $[x \sigma]$ and $[y \eta]$ share a history.

(4) Immediate from (1).

(5) Immediate from (2).

Openness is great again The construction has a desired consequence concerning openness vs. settledness. If Future $([y \eta]) \neq \emptyset$, then it is open in the sense that there is no single history that contains it wholly. In contrast, for any $[y \eta]$, Past $([y \eta])$ is settled in the sense that it is a subset of a history (typically, of more than one history). Finally, settledness of Present([yy]) is more tricky, as it depends on the details of the model. These claims are stated more precisely and then proven in the facts below.

Fact 7 (1) If Future $([y \eta]) \neq \varnothing$, then there are $[x \sigma],\left[x \sigma^{\prime}\right] \in$ Future $([y \eta])$ such that $[x \sigma] \neq\left[x \sigma^{\prime}\right]$.

(2) For every $[x \sigma] \in \operatorname{Past}([y \eta]):[x \sigma]=[x \eta]$.

Proof (1) Let Future $([y \eta]) \neq \emptyset$. By Fact 6 (4) and since histories are upward directed, there is $[x \sigma] \in$ Future $([y \eta])$ s.t. $y<_{M} x$. By clause (1)-(3) of Definition 5 there are witnesses $\sigma^{\prime} \in \Sigma, \sigma \neq \sigma^{\prime}, z \in \Re^{4}, z<_{M} x$, and atomic formula $A$ such that ( $\dagger$ ) $[z \sigma] \models A$ and $\left[z \sigma^{\prime}\right] \models \neg A$ and $[y \eta]=[y \sigma]=\left[y \sigma^{\prime}\right]$. Since $y<_{M} x, H_{x \sigma^{\prime}} \subseteq H_{y \sigma^{\prime}}$, and since $\left[y \sigma^{\prime}\right]=[y \eta]$, clause (4), i.e., $H_{x \sigma^{\prime}} \subseteq H_{y \eta}$ follows. Thus, we got $\left[x \sigma^{\prime}\right] \in$ Future $([y \eta])$. By $(\dagger)$, there is $c \in S_{\sigma, \sigma^{\prime}}$ such that $c<_{M} z$ and hence $c<_{M} x$. It follows that $[x \sigma] \neq\left[x \sigma^{\prime}\right]$.

(2) Clearly, the implication holds if Past $([y \eta])=\emptyset$. Otherwise, by Fact 6 (2) for any $[x \sigma] \in \operatorname{Past}([y \eta]), \eta \in H_{x \sigma}$, and hence $[x \sigma]=[x \eta]$. 
To comment on the proof of clause (1), note that a region of the Future of $e$ that is above $e$ is always open; yet there might be other regions of the Future of $e$, and they may fail to be open. We turn to the Present next:

Fact 8 There are MBS's in which (1) events have open Presents and (2) the relation of being co-present with a given event is not transitive.

Proof (1) A sought-for MBS is depicted at the bottom of Fig. 1. It has two space-like related binary splitting points $c$ and $c^{\prime}$ and four histories, labelled by $\sigma, \eta, \tau$, and $\gamma$. The splittings are given by $c \in S_{\sigma \tau} \cap S_{\sigma \gamma} \cap S_{\eta \tau} \cap S_{\eta \gamma}$ and $c^{\prime} \in S_{\sigma \eta} \cap S_{\sigma \gamma} \cap S_{\tau \eta} \cap S_{\tau \gamma}$. Pick $x, y \in \Re^{2}$ such that $x>c$ but $x \ngtr c^{\prime}$ and $y \ngtr c$ but $y>c^{\prime}$. Consider then $[x \sigma],[x \tau]$ and $[y \tau]$. By the splittings we have $(\dagger) H_{x \sigma}=\{\sigma, \eta\}, H_{x \tau}=\{\tau, \gamma\}$, and $H_{y \tau}=H_{y \sigma}=\{\sigma, \tau\}$. So $[x \sigma]$ and $[y \tau]$ share a history $(\sigma)$ and $[x \tau]$ and $[y \tau]$ share a history $(\tau) .{ }^{18}$ Also, $H_{x \sigma} \backslash H_{y \sigma} \neq \emptyset, H_{y \sigma} \backslash H_{x \sigma} \neq \emptyset, H_{x \tau} \backslash H_{y \tau} \neq \emptyset$, and $H_{y \tau} \backslash H_{x \tau} \neq \varnothing$. It follows that $[x \sigma],[x \tau] \in \operatorname{Present}([y \tau])$. On the other hand, $x>c \in S_{\sigma \tau}$ implies $[x \sigma] \neq[x \tau]$, which proves that $\operatorname{Present}([y \tau])$ is open.

(2) The same MBS is a witness for the second claim. The co-presence is defined by: $C P\left(e, e^{\prime}\right)$ iff $e \in \operatorname{Present}\left(e^{\prime}\right)$. By Fact 6 (3), CP is reflexive and symmetric. So we have $C P([x \sigma],[y \tau])$ and $C P([y \tau],[x \tau])$. But by $(\dagger)$ above, $[x \sigma]$ and $[x \tau]$ do not share a history, so they are not co-present.

Note that our witness for the openness of future and non-transitivity of co-presence is based on a pair of SLR splitting points, with the splitting working "combinatorially". That is, two outcomes split at each splitting points, and then we have four histories. It takes little reflection to see that this pattern of splitting always leads to the openness of future and non-transitivity of co-presence. A remedy is to postulate that splitting does not happen combinatorially. We return to this subject on p. 28

Is the tripartite division observed? The way we defined the three notions, Past, Present, and Future of event $[y \eta]$, they divide any history to which $[y \eta]$ belongs. That is, for any history $h_{\sigma}=\left\{[x \sigma] \mid x \in \mathfrak{R}^{4}\right\}, \sigma \in \Sigma$, and such that $[y \eta]=[y \sigma] \in h_{\sigma}$,

$$
h_{\sigma}=(\operatorname{Past}([y \eta]) \cup \operatorname{Present}([y \eta]) \cup \operatorname{Future}([y \eta])) \cap h_{\sigma} .
$$

Since Future $([y \eta])$, if non-empty, is not fully contained in any history (and Present $([y \eta])$ might happen not to be fully contained in any history), we need to have an intersection with $h_{\sigma}$ on the RHS of the above equation. Note however that in some cases the division might be into fewer sets than three, since Future, or Past, or both might be empty (see Sect. 6). On the other hand, Present $([y \eta]) \neq \varnothing$ for every $[y \eta]$, since $[y \eta] \in \operatorname{Present}([y \eta])$, see Fact 6 (3). Thus, the tripartite division can be degenerate, but it holds (degenerate or not). Another weirdness is that Past and Future of a given event can overlap-see Sect. 6 .

Invariance The Past, Present, and Future of a given event are defined in terms of precausal ordering $\leqslant$ and set-theoretical operations on it. This ordering, if restricted to a

\footnotetext{
18 For brevity's sake, we write "in history $\sigma$ ", though to be correct, it should be "in the history labelled by $\sigma ’$
} 
single history, yields the Minkowski ordering $\leqslant_{M}$, see Eq. 8, which is invariant with respect to automorphisms of Minkowski space-time. Thus, the regions of histories that our definitions pick out are invariant with respect to these automorphisms.

Transitivity of co-presence-modal correlations to the rescue? Clearly, copresence is reflexive and symmetric [(each by Fact 6 (3)]. However, it may fail to be transitive, which we showed in Fact 8 . The proof was based on disjunct (ii) of Fact 6 (3), which requires non-empty differences of the sets of appropriate labels. Observe, however, that if co-presence is based on identity of sets of labels (which is disjunct (i) of the same Fact), transitivity obtains since obviously identity is transitive. This brings in the question: under what condition the nice disjunct (i) $H_{x \sigma}=H_{y \sigma}$ rather than the troublesome disjunct (ii) of Fact 6 (3) obtains?

To unpack (i), it means that for every $\gamma \in \Sigma, x \in R_{\sigma \gamma}$ iff $y \in R_{\sigma \gamma}$ (here we already incorporated the condition that $[x \sigma]$ and $[y \eta]$ share a history, $\sigma$ ). This is further equivalent to the claim that for any $\gamma, \exists c \in S_{\sigma \gamma} c<_{M} x$ iff $\exists c^{\prime} \in S_{\sigma \gamma} c^{\prime}<_{M} y$. This condition is trivially satisfied if histories split from $\sigma$ in the common past of $x$ and $y$ rather than in the past of one of them only. It has a bite if $\sigma$ splits from histories $\gamma$ and $\eta$ at two space-like related points $c \in S_{\sigma \gamma}$ and $c^{\prime} \in S_{\sigma \eta}$ only, where $c<_{M} x$ but $c \nless_{M} y$, and $c^{\prime}<_{M} y$ but $c^{\prime} \nless_{M} x$. Then the condition requires that exactly the same histories must split at $c$ and $c^{\prime}$. Thus, $c \in S_{\sigma \eta}$ and $c^{\prime} \in S_{\sigma \gamma}$ as well. This coordinated splitting is in contrast to the MBS with "combinatorial" splitting depicted at the bottom of Fig. 1, where, for instance, $\sigma$ and $\tau$ split at $c$, but not at $c^{\prime}$.

Somewhat worryingly, such coordinated splittings are reminiscent of EPR correlations, modally understood. On this understanding, although each of the two space-like related measurements has two possible outcomes, say + and - , some joint combinations of these outcomes, say ++ , or -- , are not possible. ${ }^{19}$ Such modal correlations, known also as "modal funny business", MFB, have been analyzed in BST literature, in particular in the context of quantum experiments, cf. Belnap (2002, 2003), Müller et al. (2008), and references therein.

Since in the condition above the quantification is over all histories $\gamma$, in order to enforce the transitivity of co-presence in the whole MBS, one needs to assume the following global condition:

Definition 9 (MFB) For every $\sigma, \gamma, \eta \in \Sigma$ and every $c, c^{\prime} \in \mathfrak{R}^{4}$ :

$$
\text { if } c \in S_{\sigma \gamma}, c^{\prime} \in S_{\sigma \eta} \text { and } c S L R c^{\prime} \text {, then } c^{\prime} \in S_{\sigma \gamma} \text {. }
$$

We prove now that the condition of MFB does some useful work:

Fact 10 For any event $[y \eta]$ in an MBS that satisfies MFB, (1) Present ([y $]]$ ) is settled and (2) co-presence is transitive.

Proof (1) From the assumption that there are distinct events $[x \sigma],\left[x \sigma^{\prime}\right] \in$ Present $([y \eta])$ we derive a contradiction with MFB. By Fact 6 (3), there are $\gamma, \gamma^{\prime} \in \Sigma$

19 Observe that missing joint outcomes need not be understood in modal terms, as the impossibility of certain histories. A more standard move, as suggested by quantum mechanics, is to assign zero probability to such (seemingly) impossible results. 
such that $[x \sigma]=[x \gamma],\left[x \sigma^{\prime}\right]=\left[x \gamma^{\prime}\right],[x \gamma] \neq\left[x \gamma^{\prime}\right]$, and $(\dagger)[y \eta]=[y \gamma]=\left[y \gamma^{\prime}\right]$. Hence $x \notin R_{\gamma \gamma^{\prime}}$ but $y \in R_{\gamma \gamma^{\prime}}$. There is thus $c \in S_{\gamma \gamma^{\prime}}$ such that $c<_{M} x$ but $c \nless_{M} y$. Further, since $\gamma^{\prime} \in H_{y \gamma}$ but $\gamma^{\prime} \notin H_{x \gamma}, H_{y \gamma} \neq H_{x \gamma}$. Thus, condition (i) of Fact 6 (3) is not satisfied, so condition (ii) must hold, so in particular we must have $H_{x \gamma} \backslash H_{y \gamma} \neq \emptyset$. Thus, for some $\alpha \in \Sigma:[y \alpha] \neq[y \gamma]$ but $[x \alpha]=[x \gamma]$. There is thus $c^{\prime} \in S_{\alpha \gamma}$ such that $c^{\prime}<_{M} y$ but $c^{\prime} \nless_{M} x$. It cannot be that $c$ and $c^{\prime}$ are comparable, as it implies $c^{\prime}<_{M} x$ or $c<_{M} y$. Hence $c$ SLR $c^{\prime}$. As the premises of MFB are satisfied, it follows that $c^{\prime} \in S_{\gamma \gamma^{\prime}}$. Since $c^{\prime}<_{M} y$, we get that $[y \gamma] \neq\left[y \gamma^{\prime}\right]$, which contradicts ( $\left.\dagger\right)$. We thus derived a contradiction from the assumption that Present $([y \eta])$ is open, which proves that it is settled.

(2) Recall that co-presence is symmetric. Let us thus suppose that (†) $[x \sigma],\left[x^{\prime} \sigma^{\prime}\right] \in$ $\operatorname{Present}([y \eta])$. We argue that $\left[x^{\prime} \sigma^{\prime}\right] \in \operatorname{Present}([x \sigma])$. As we just proved, Present is settled, so there is $\alpha \in \Sigma$ such that (†) $[x \sigma]=[x \alpha],\left[x^{\prime} \sigma^{\prime}\right]=\left[x^{\prime} \alpha\right]$, and $[y \eta]=[y \alpha]$. By the argument like in the proof above, given MFB, condition (ii) of Fact 6 (3) cannot be satisfied. That is, it is not true that $\left(H_{x \alpha} \backslash H_{y \alpha} \neq \emptyset\right.$ and $\left.H_{y \alpha} \backslash H_{x \alpha} \neq \emptyset\right)$, and it is not true that $\left(H_{x^{\prime} \alpha} \backslash H_{y \alpha} \neq \emptyset\right.$ and $\left.H_{y \alpha} \backslash H_{x^{\prime} \alpha} \neq \emptyset\right)$. Thus, since ( $\left.\dagger\right)$ holds, condition (i) of Fact 6 (3) must be satisfied, i.e., $H_{x \alpha}=H_{y \alpha}$ and $H_{x^{\prime} \alpha}=H_{y \alpha}$. Thus, $H_{x^{\prime} \alpha}=H_{x \alpha}$. This together with ( $\$)$ shows that $\left[x \sigma^{\prime}\right] \in \operatorname{Present}([x \sigma])$.

Separation of past, present, and future from causal cones By our Definition 5, it may transpire that the Future of event $e$ is larger than the future light cone of $e$. Similarly, Past of event $e$ may happen to be larger that the past light cone of $e$. These results reflect the absence of a causal-order condition in our explication of Future, i.e., we do not require that $e<f$ for $f$ being in the future of $e$. This is because we want Past, Present, and Future to be defined in terms of modal notions only, which is indeed achieved (see Fact 6). Also, we want to have a possibly thin Present, which calls for making Past and Future possibly large.

A more general observation is that tenses work differently than the pre-causal ordering $\leqslant$. It might happen that an event belongs to the Present of a given event, although it is pre-causally before (or after) it. This has further counter-intuitive consequences. For instance, consider three pre-causally related events $e<e^{\prime}<e^{\prime \prime}$ such that in the history to which they all belong there is just one choice point $c$, which is located between $e$ and $e^{\prime}$. Our framework then gives the verdict that $e^{\prime}$ and $e^{\prime \prime}$ belong to the Future of $e$, but $e^{\prime \prime}$ belongs to the Present of $e^{\prime}$ rather than to the Future of $e^{\prime} .{ }^{20}$ As we may make the temporal distance (in terms of metric time) between $e^{\prime}$ and $e^{\prime \prime}$ arbitrarily large, the worry is that $e^{\prime \prime}$ "comes to being well before its time". One may try to alleviate this worry by interpreting the succession of causally extended presents as a granular way of becoming. On this reading, the fact that two temporally remote events are co-present means that they come to being at the same stage of becoming. Having said so, I concede that the separation of tenses from causal and temporal relations $i s$ counter-intuitive. To make things worse, not every model allows for the succession of presents - see above on the transitivity of co-presence.

What is it really like? Since the Past, Present and Future of events depends heavily on where, and how often, alternative histories split, a premonition of whether, and if so,

${ }^{20}$ For this example I am grateful to an anonymous reviewer for this journal. 

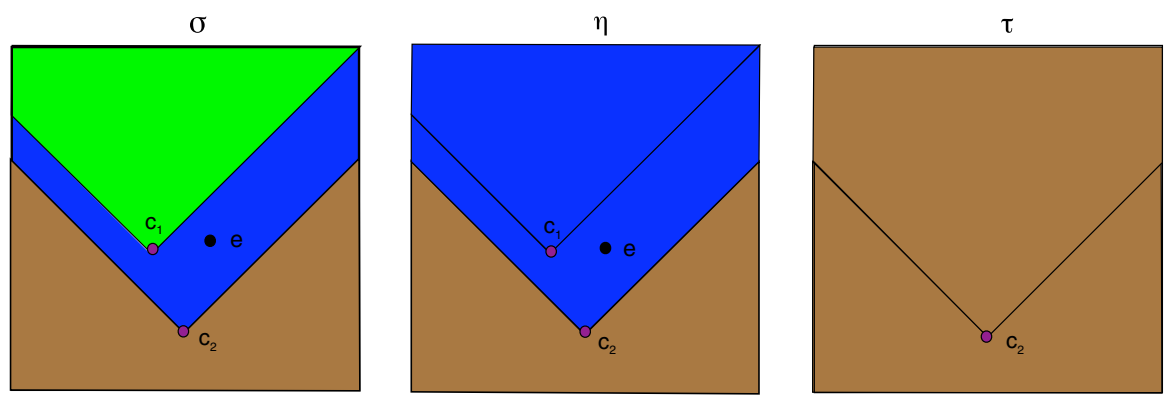

Fig. 2 The past, the present, and the future of $e=[x \sigma]$

how splitting happens in our world is highly interesting. As this is a large topic, two remarks must suffice here. On a standard understanding of radioactivity, a radioactive particle existing without decay for a time being makes for a continuous set of splitting points. The existence of modal correlations, EPR-style, is more controversial, because the "lacking histories" can be modeled probabilistically rather than modally, by having only tiny, or just null, probabilities assigned.

Going Beyond Minkowski In this paper we have restricted ourselves to analyzing Past, Present, and Future in a modal framework in which histories are isomorphic to Minkowski space-time. One might naturally wonder if this analysis can carry over to other space-times. The answer is in the affirmative-as long as the space-times (or rather, histories based on these space-times) satisfy BST axioms. Although the axioms are not too restrictive, as for instance they permit many space-times of General Relativity, they imply asymmetry (i.e., if $e<e^{\prime}$, then $\neg\left(e^{\prime}<e\right)$ ), which means that they exclude space-times with causal loops.

\section{What do the past, present, and future look like?}

We will now apply Definitions 5 and 2, and Fact 6 to some selected MBS's to visualize what the future, the present and the past of a given event are. The direction of this little survey is from "nice" to "weird" examples.

Two time-like splitting points Consider first an MBS with three histories, i.e. $\Sigma=\{\sigma, \eta, \tau\}$, in which the proper property attribution yields two splitting points $c_{1}, c_{2} \in \mathfrak{R}^{4}$ such that $c_{2}<_{M} c_{1}$ and $S_{\sigma \eta}=\left\{c_{1}\right\}$ and $S_{\sigma \tau}=S_{\eta \tau}=\left\{c_{2}\right\}$. That is, $\eta$ and $\sigma$ split from $\tau$ at $c_{2}$, and then $\eta$ splits from $\tau$ at $c_{1}$. Figure 2 represents these three histories as squares with a common bottom region. To explain the shadow convention, a difference in shadow indicates that the corresponding regions are not to be identified (but that does not imply that every two corresponding points have different properties).

Now pick any event $e:=[x \sigma]$ that is above $c_{2}$ but not above $c_{1}, c_{2}<_{M} x$ and $x \ngtr_{M} c_{1}$ and ask: (1) What is the future of $e$ ? (2) What is its past? (3) And what is its present? Fact 6 yields the following verdicts: 
Fig. 3 History $\left\{[x \sigma] \mid x \in \mathfrak{R}^{4}\right\}$ with four splitting points $c_{1}, \ldots, c_{4}$. The present of event $e=[x \sigma]$ is indicated by the shaded area

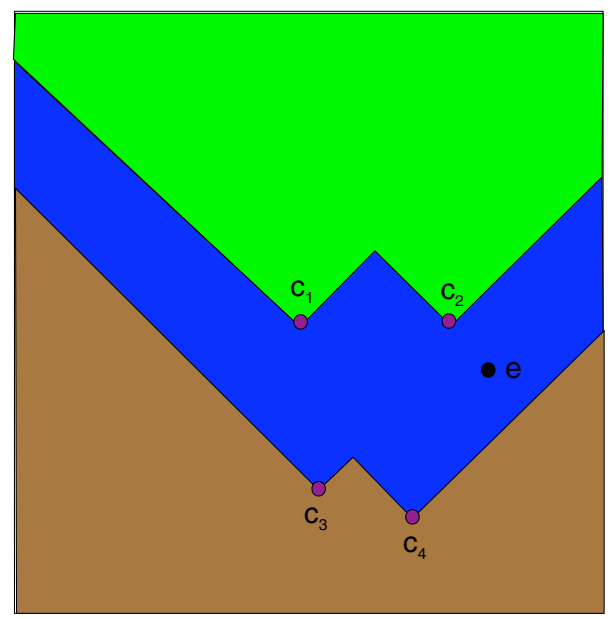

$\sigma$

1. The future of $e=[x \sigma]$ is the set of events that are strictly above $\left[c_{1} \sigma\right]$ :

Future $([x \sigma])=\left\{[z \gamma] \mid c_{1}<_{M} z \wedge \gamma \in\{\sigma, \eta\}\right\}$. Note that this region is the union of two future light cones of $c_{1}$, in histories labeled by $\sigma$ and $\eta$. The light cones include boundaries but exclude $e$. And the future of $e$ is above $c_{1}$ rather than above $e$.

2. The past of $e=[x \sigma]$ is the set of events that are in history $\sigma$ and not strictly above $\left[c_{2} \sigma\right]: \operatorname{Past}([x \sigma])=\left\{[z \sigma] \mid c_{2} \nless_{M} z\right\}$. In contrast to the future, the past is shared by all the three histories.

3. The present of $e=[x \sigma]$ is the set of events in history $\sigma$ and "between" $c_{2}$ and $c_{1}$ in the sense: $\operatorname{Present}([x \sigma])=\left\{[z \sigma] \mid c_{2}<_{M} z \wedge c_{1} \nless_{M} z\right\}$. The present of $e$ is shared by the two histories to which $e$ belongs.

Note that the present of $e=[x \sigma]$ turns out to be a spatially extended and temporally thick collection of events. Its temporal thickness depends on the Lorentz interval of the (time-like) vector $c_{1} c_{2}$. Moreover, in this model co-presence is transitive as all sets of splitting points contain just one element, so condition MFB is automatically satisfied.

Four splitting points, layered in two SLR pairs Consider an MBS with three histories, i.e., $\Sigma=\{\sigma, \eta, \tau\}$, with $S_{\sigma \eta}=\left\{c_{1}, c_{2}\right\}$ and $S_{\sigma \tau}=S_{\eta, \tau}=\left\{c_{3}, c_{4}\right\}$, where $c_{3}<_{M} c_{1}$ and $c_{4}<_{M} c_{2}$-see Fig. 3. Next, pick an arbitrary event [xo], with $x$ sliced between two pairs of splitting points, that is, $\left(c_{3}<_{M} x\right.$ or $\left.c_{4}<_{M} x\right)$ and $\left(c_{1} \nless_{M} x\right.$ and $c_{2} \nless_{M} x$ ). Applying Fact 6 we get this result:

$$
\operatorname{Present}([x \sigma])=\left\{[y \sigma] \mid\left(y>_{M} c_{3} \vee y>_{M} c_{4}\right) \wedge\left(y \ngtr_{M} c_{1} \wedge y \ngtr_{M} c_{2}\right)\right\} .
$$

Thus, the present of $[x \sigma]$ turns out to have the shape of a thick letter $W$. Note that by adding more splitting points to $S_{\sigma \eta}$ and to $S_{\sigma \tau}$ we obtain as Present a "generalized" letter $W$, with more top corners and more bottom corners. And by making smaller the separation between $S_{\sigma \eta}$ and $S_{\sigma \tau}$ we can make the generalized $W$ arbitrarily thin. We 
can make it extend through the whole history as well. Yet beware: each construction of this sort requires EPR-like modal correlations, that is, the satisfaction of condition MFB. Thus, we may arbitrarily closely approximate the Present as an infinitely thin maximal space-like hyper-surface, but at the price of a massive EPR-like modal correlations.

Extreme cases (i) Let us first focus on an MBS with two histories $\sigma$ and $\eta$, which split at a single point $c$, so $\{c\}=S_{\sigma \eta}$. For $x \ngtr c$ (which includes $\left.x=c\right)$, Present $([x \sigma])=$ $\{[y \sigma] \mid y \ngtr c\}$ and Future $([x \sigma])=\{[y \sigma] \mid y>c\}$. Note that $\operatorname{Past}([x \sigma])=\emptyset$. The situation is symmetrical for $x>c$ : Present $([x \sigma])=\{[y \sigma] \mid y>c\}$, Past $([x \sigma])=$ $\{[y \sigma] \mid y \ngtr c\}$, and Future $([x \sigma])=\emptyset$.

(ii) Next consider an MBS with just one history $\sigma$. This is a depiction of global determinism. In this MBS, for every event, its past as well as its future are empty, from which it follows that for every event, its present is the entire history. We thus have a block universe, indeed. This is a controversial verdict as the popular intuition sees a non-trivial past, present, and future in a deterministic word. For a representative statement of this intuition, see Gale (1963).

(iii) At the other extreme, if a history splits at every point with some other history, that is, if $\forall x \in \Re^{4} \exists \eta \in \Sigma x \in S_{\sigma \eta}$ for some $\sigma \in \Sigma$, then every event in history $\sigma$ has non-trivial past, present, and future. What do they look like, depends on the details of the model, in particular whether it satisfies MFB. MFB may be used to construct sets $S_{\sigma \eta}$ as maximal space-like surfaces that continuously foliate history $\sigma$. Each surface of this kind then is the Present of every event that belongs to it. If MFB is not satisfied, the shape of an event's present depends on the particular way in which the condition fails. In particular, if all splittings are combinatorial, for each event $e$ its Past and Present contain all events space-like related to $e$ (so they overlap), and Present $(e)$ is identical to $e$ itself.

\section{Conclusions}

We have investigated a modal explication of the notions of past, present and future, one constructed on the idea that the past is settled, but the future is open. In choosing a link between future and contingency, we decided for a possibly weak association encapsulated by $\mathbf{S}$. We then used a rigorous framework of BST and MBS to write down our definitions, and prove some facts about an event's past, present, and future if the underlying space-time is Minkowski.

A salient feature of this construction is that the shape of the present (past and future) of event $e$ from history $h$ depends on the location of the splitting points of $h$, understood as chancy events in $h$. These locations depend in turn on the localization of qualitative differences between $h$ and histories alternative to it. The shapes of the present vary wildly, from the entire history in deterministic models, to a maximal space-like surface of Minkowskian history in a model with massive EPR-like modal correlations, or to a single event in models in which there are no such correlations but every event is chancy. Shapes of the past (the future) vary wildly as well. 
Perhaps the most surprising aspect of our construction is that the condition of MFB (which generalizes EPR correlations, by reading them modally) do useful work. It ensures that co-presence is a transitive relation and that the present of any event is settled (compare Facts 8 and 10). It can be used to foliate a history into the succession of presents. Thus, with MFB our construction may deliver tenses that agree with everyday's intuitions.

To finish the paper, we would like to take stock of what the proposed construction achieves. We will therefore go through the list of features of manifest time given in Sect. 1, to check which are satisfied in our construction, and which are not.

To begin with the modal aspect, past, present, and future supervene on modal notions alone, that is, on relations between sets of histories to which events belongsee Fact 6. Next, if an event's past, present, and future are all non-empty, they provide a tripartite division of each history that contains the event. But there are models in which an event's past, or future, or both are empty, so then the division is less than tripartite. An event's future, if non-empty, is open, whereas its past is settled in the sense that no history contains the whole (non-empty) future, whereas an event's past is fully comprised in some history. Further, the present is settled provided that the model satisfies condition MFB.

There are, however, a few features of manifest time that our construction may violate. Emphatically, tenses work differently than metric time and the pre-causal relation that generalizes the ordering defined by light-cones. Most conspicuously, special cases aside, an event's present is not achronal. That entails that the past, present, and future of our construction fly in the face of grammatical tenses in English. One may truly say at event $e$ that $e^{\prime}$ will happen, whereas $e^{\prime}$ belongs to $e$ 's present. Another flaw is that generally co-presence is not transitive in models without the MFB condition. Next, in some models, an event's past and future overlap. Furthermore, it is not always true that the presents of different events either agree or do not overlap. This is because, without the MFB condition, co-present SLR events may have different futures. This endangers the idea of the flow of time as the succession of one present following after another. Turning to the next feature, an event's present is not always global, since it can be identical to the event itself, or have a $V$-shape or a $W$-shape to be seen in Figs. 2 and 3, respectively, which can be avoided. However, models with an event's present identified with a maximal space-like surface are also possible, and in Minkowskian history they cannot be avoided by an object that exists sufficiently long. No matter whether the global character of an event's present is satisfied, the present cannot be repeated. Finally, to recall, modeling the flow of time is outside the scope of this project.

Having seen the pluses and minuses of modally defined past, present, and future, we leave it to the reader to decide whether these are viable concepts. We also invite the reader to play with our definitions of past, present, and future to attempt to deliver a more intuitive, but still modally introduced, concept of tenses. We nevertheless believe that our definitions achieve a good balance between, on the one hand, defining tenses in terms of modalities, and saving intuitions about tenses, on the other. 
Open Access This article is distributed under the terms of the Creative Commons Attribution 4.0 International License (http://creativecommons.org/licenses/by/4.0/), which permits unrestricted use, distribution, and reproduction in any medium, provided you give appropriate credit to the original author(s) and the source, provide a link to the Creative Commons license, and indicate if changes were made.

\section{Appendix: Postulates of BST1992}

A BST 1992 structure is a pair $\langle W, \leqslant\rangle$ that fulfills the following conditions:

1. $W$ is a non-empty set of possible point events.

2 . $\leqslant$ is a partial ordering denoting a pre-causal relation on $W$.

3. The ordering $<$ is dense;

$$
\forall x, y \in W[x<y \Rightarrow \exists z \in W[x<z<y]] .
$$

4. The ordering contains infima for all lower bounded chains: If $l \subseteq W$ is a chain that has a lower bound (for some $e \in W, e \leqslant l$ ), then $l$ has a unique greatest lower bound $\inf l$ :

$$
\forall x[x \leqslant l \Rightarrow x \leqslant \inf l] .
$$

5. The ordering contains history-relative suprema for all upper bounded chains: If $l \subseteq W$ is a chain with an upper bound (for some $e \in W, l \leqslant e$ ), and $h$ is a history for which $l \subseteq h$, then $l$ has a unique smallest upper bound $\sup _{h} l$ in $h$ :

$$
\forall x\left[(x \in h \wedge l \leqslant x) \Rightarrow \sup _{h} l \leqslant x\right]
$$

6. Weiner's postulate: Let $l, l^{\prime} \subseteq h_{1} \cap h_{2}$ be upper bounded chains in histories $h_{1}$ and $h_{2}$. Then the order of the suprema in these histories is the same:

$$
\sup _{h_{1}} l \leqslant \sup _{h_{1}} l^{\prime} \quad \text { iff } \quad \sup _{h_{2}} l \leqslant \sup _{h_{2}} l^{\prime} .
$$

7. Prior Choice Principle: If a chain $I$ is lower bounded, and for histories $h_{1}, h_{2}$, $I \subseteq h_{1}$ and $I \cap h_{2}=\emptyset$, then there is an event $c$ that is maximal in the intersection $h_{1} \cap h_{2}$ and such that $c<I$.

A history in $\langle W, \leqslant\rangle$ is defined as a maximal upward directed subset of $W .{ }^{21}$ Prior Choice Principle implies that a typical BST1992 structure is not locally Euclidean, which might be troublesome. There is an alternative but computationally more demanding formulation of the Principle, which saves local Euclidicity by requiring $c$ to be a minimal element in the histories' difference, $h_{1} \backslash h_{2}$.

${ }^{21} A \subseteq W$ is called "upward directed" if for any $x, y \in A$ there is some $z \in A$ such that $x \leqslant z$ and $y \leqslant z$. Maximal upward directed subsets of $W$ can be proved to exists (by using the Zorn-Kuratowski lemma). 


\section{References}

Belnap, N. (1992). Branching space-time. Synthese, 92, 385-434. 'Postprint' archived at PhilSci Archive. http://philsci-archive.pitt.edu/archive/00001003.

Belnap, N. (2002). EPR-like "funny business" in the theory of branching space-times. In T. Placek \& J. Butterfield (Eds.), Nonlocality and modality (pp. 293-315). Dordrecht: Kluwer Academic Publisher.

Belnap, N. (2003). No-common-cause EPR-like funny business in branching space-times. Philosophical Studies, 114, 199-221.

Belnap, N. (2005). A theory of causation: Causae causantes (originating causes) as inus conditions in branching space-times. British Journal for the Philosophy of Science, 56, 221-253.

Belnap, N. (2007). An indeterminist view on the parameters of truth. In T. Müller (Ed.), Philosophie der Zeit (pp. 87-113). Frankfurt: Klostermann.

Belnap, N., \& Müller, T. (2014). BH-CIFOL: Case-intensional first order logic. Journal of Philosophical Logic, 43(5), 835-866.

Callender, C. (2017). What makes time special. Oxford: Oxford University Press.

Dieks, D. (1988). Special relativity and the flow of time. Philosophy of Science, 55(3), 456-460.

Dorato, M. (2008). Putnam on time and special relativity. European Journal Analytic Philosophy, 4(2), $51-70$.

Eddington, A. S. (1949). The nature of physical world. Cambridge: Cambridge University Press.

Eddington, A. S. (1953). Space, time, gravitation. Cambridge: Cambridge University Press.

Ellis, G. F. R. (2006). Physics in the real universe: Time and space-time. General Relativity and Gravitation, 38, 1797-1824.

Gale, R. M. (1963). Some metaphysical statements about time. The Journal of Philosophy, 60(9), $225-237$.

Lewis, D. K. (1986). Philosophical papers: Volume II. Oxford: Oxford University Press.

Malament, D. (1977). Causal theories of time and the conventionality of simultaneity. Nous, 11(3), 293-300.

Müller, T. (2002). Branching space-time, modal logic and the counterfactual conditional. In T. Placek \& J. Butterfield (Eds.), Nonlocality and modality, NATO science series (pp. 273-291). Dordrecht: Kluwer Academic Publisher.

Müller, T. (2005). Probability theory and causation: A branching space-times analysis. British Journal for the Philosophy of Science, 56(3), 487-520.

Müller, T. (2006). On the problem of defining the present in special relativity: A challenge for tense logic. In F. Stadler \& M. Stöltzner (Eds.), Time and history. Proceedings of the 28. International Ludwig Wittgenstein symposium, Kirchberg am Wechsel, Austria 2005 (pp. 441-458). Frankfurt: Ontos Verlag.

Müller, T., Belnap, N., \& Kishida, K. (2008). Funny business in branching space-times: Infinite modal correlations. Synthese, 164(1), 141-159.

Placek, T. (2011). A locus for 'now'. In D. Dieks, et al. (Eds.), Explanation, prediction, and confirmation: New trends and old ones reconsidered (pp. 339-410). Dordrecht: Springer.

Placek, T., \& Belnap, N. (2012). Indeterminism is a modal notion. Synthese, 187(2), 441-469.

Prior, A. (1967). Past, present, and future. Oxford: Oxford University Press.

Putnam, H. (1967). Time and physical geometry. The Journal of Philosophy, 64(8), 240-247.

Rakić, N. (1997). Past, present, future, and special relativity. The British Journal for the Philosophy of Science, 48(2), 257-280.

Rietdijk, C. W. (1966). A rigorous proof of determinism derived from the special theory of relativity. Philosophy of Science, 33(4), 341-344.

Stein, H. (1991). On relativity theory and openness of the future. Philosophy of Science, 58(2), 147-167.

Thomason, R. H. (1970). Indeterminist time and truth-value gaps. Theoria, 36, 264-281.

van Benthem, J. (1991). The logic of time. Dordrecht: Kluwer AP.

Whitrow, G. J. (1961). The natural philosophy of time. London: Thomas Nelson and Sons Ltd.

Wroński, L., \& Placek, T. (2009). On Minkowskian branching structures. Studies in History and Philosophy of Modern Physics, 40, 251-258.

Publisher's Note Springer Nature remains neutral with regard to jurisdictional claims in published maps and institutional affiliations. 